\title{
Young children's developing ability to integrate gestural and emotional cues. ${ }^{[1]}$
}

Running Title: Integrating gestural and emotional cues.

\author{
Authors: \\ Gregor Kachel ${ }^{1,2}$ \\ David Hardecker ${ }^{1}$ \\ Manuel Bohn $n^{1,2,3}$ \\ Affiliations: \\ ${ }^{1}$ Leipzig University \\ ${ }^{2}$ Max-Planck-Institute for Evolutionary Anthropology, Leipzig \\ ${ }^{3}$ Stanford University
}

\section{Acknowledgements:}

We would like to thank Anja Ibes for data collection, Jana Jurkat for administrative support, Jana Mahler for reliability coding, Sylvio Tüpke for the photographs, and Linda Oña for helping with the FACS-Coding. We are grateful to Michael Tomasello for his support throughout the study. Finally, we thank all the children and their parents for participating. Manuel Bohn was supported by the European Union's Horizon 2020 research and innovation programme under the Marie Sklodowska-Curie grant agreement No 749229.

The authors declare no conflict of interest.

[1] Address for correspondence: Gregor Kachel, Faculty of Educational Sciences, Leipzig University, Dittrichring 5-7, 04109 Leipzig, Germany. Email: gregor.kachel@gmail.com 


\title{
Young children's developing ability to integrate gestural and emotional cues.
}

Running Title: Integrating gestural and emotional cues

\section{Date of submission:}

$18 / 02 / 2020$

\begin{abstract}
:
In three studies, children between 22 and 46 months of age $(N=180)$ had to integrate pointing gestures or gaze cues with positive and negative facial expressions to succeed in an object-choice-task. In a between-subjects-design, finding the toy required children to either choose (positive expression) or avoid (negative expression) the indicated target. Study 1 showed that 22-month-olds are better at integrating positive compared to negative facial expressions with pointing gestures. Study 2 tracked the integration of negative expressions and pointing across development, finding an unexpected, u-shaped trajectory with group-level success only at 46 months. Study 3 showed that already 34-month-olds succeeded when pointing was replaced with communicative gaze. Findings show that communicative cues need to be studied in conjunction to draw an ecologically valid picture of communicative development, and that pointing - beyond indexing - has an affirmative meaning for young children.
\end{abstract}

Keywords: Social referencing; pointing; gaze; communication; emotion understanding; negation

The authors declare no conflict of interest. 


\section{Introduction}

Human communication is inherently multimodal and requires the integration of different types of information to recover a speaker's intended meaning. Non-verbal cues such as gestures and facial expressions play a crucial role in children's earliest interactions, especially in the acquisition of the conventional semantics of words (Colonnesi, Stams, Kosten \& Noom, 2010; Tomasello, 2003; 2008). Much like words in spoken language, gestures and emotional displays can be combined in productive ways and modify each others meaning systematically. A pointing gesture, for instance, may invite very different actions when combined with either positive or negative facial expressions. If you ask your friend for advice on what kind of dish to order and she replies by pointing to the plate in front of her while smiling, you might feel encouraged to get the same. You would probably arrive at a different conclusion if the same pointing gesture was accompanied by a disgusted facial expression. Here, you should specifically avoid this option.

Young children encounter such combinations of deictic reference with negative valence not only when enquiring other's preferences, but on a daily basis in the form of prohibitory and safety rules (Dunn \& Munn, 1987; Kopp, 1982; Smetana, Kochanska, \& Chuang, 2000). Whenever caregivers impose restrictions on where to go or what to touch, they need to highlight the respective object or place via some sort of reference and then add meaning to it with emotions expressed in their tone of voice and facial expression - especially so with young children that are in the middle of acquiring language in the first place.

In the studies presented here, we investigate the development of children's ability to use referential and emotional cues when making inferences about a speaker's intended meaning. Specifically, we tested how children respond to combinations of pointing gestures or gaze with salient positive or negative facial expressions. We operationalized the task much 
like in the food example above but strictly restrict children's alternatives to scaffold their inferences. In an object-choice task with two hiding places, we establish as common ground that one of two hiding places is baited with a desired toy and that children's interlocutor is both knowledgeable and intent on helping them. After hiding the desired object (a marble) under one of two boxes outside the view of the child, the experimenter indicated one of the locations, accompanied by either a positive or negative facial expression. To succeed in the case of a positive expression, children can simply follow the gesture and retrieve the marble. In the negative condition, however, the experimenter effectively conveys not to take the one she pointed at and that the marble is in the other hiding place. This requires children to (1) understand the emotional display, (2) follow the referential cue, (3) reason by exclusion that the target must be in the other hiding place and (4) inhibit the prepotent response of taking indicated option, which is especially challenging in the context pointing gestures (Grassmann \& Tomasello, 2010; Stengelin, Grüneisen \& Tomasello, 2018). Below, we review when each of these skills develops and highlight why the set of studies we present here does not only broaden our understanding of pragmatic development but is also relevant for current work on epistemic vigilance.

Prior to understanding caregiver's explicit instruction, infants and toddlers are to understand salient emotional states as expressed in facial expressions and vocalizations. Already at 4 months, infants discriminate discrete emotions such as happy, angry and sad when they are expressed multimodally (Montague \& Walker-Andrews, 2001; Flom \& Bahrick, 2007). At 12 months, children also begin to understand the referential dimension of emotional displays and use emotional expressions to gain information about ambiguous events or objects (Sorce, Emde, Campos \& Klinnert, 1985). For example, 12- but not 8-month-olds expect an actor who emotes positively to one of two objects to reach for this 
object subsequently (Phillips, Wellman \& Spelke, 2001). In a visual cliff scenario, Sorce et al. (1985) found that 12-month olds would halt at the visual cliff when the mother showed a fearful expression but would cross when the mother expressed interest or joy. 12- to 18-month-old children adapt their behavior towards a novel toy in line with an adult's positive or negative vocalizations when gazing at a toy (Moses, Baldwin, Rosicky \& Tidball, 2001). When a stranger enters a room, 15-month-old children look at their mother and adjust their reaction according to the mother's facial expression (positive or ignoring; Feiring, Lewis, \& Starr, 1984). When facing unusual toys, young children approach their mothers if she shows a fearful facial expression but approach the toy if she shows a joyful expression (Klinnert, 1984). One of the most comprehensive study of infants' reactions to directed emotional displays is provided by Walle and colleagues (2017) and shows that 16-, 19- and 24-month-olds react differently towards objects that were simultaneously pointed at while either expressing joy, sadness, fear, anger or disgust. While all age-groups were more generally more likely to approach an object that was highlighted with joy as opposed to disgust or anger, it is remarkable that 30 to $50 \%$ of children in all age-groups went on to explore stimuli that were pointed to and marked with disgust or fearful expressions (Walle, Reschke, Camras \& Campos, 2017).

At end of the first year, children gain competence to understand emotions and their directedness at the world, children also begin to understand ostensive cues, that is, cues that direct the attention of the child in a communicatively meaningful way. From 10 months onwards pointing gestures directionally influence infants' attention (Gredebäck, Melinder, \& Daum, 2010). Infants spontaneously start using pointing gestures to communicate with adults about two months later (Matthews, Behne, Lieven, \& Tomasello, 2012). Production of pointing goes hand in hand with comprehension (Behne, Liszkowski, Carpenter, \& 
Tomasello, 2012). Around the same age, children differentiate between intentionally produced pointing gestures and points that arise as a by-product of other actions, suggesting that infants differentiate between the signal itself and the speaker's intentions behind it (Aureli, Perucchini, \& Genco, 2009; Behne, Carpenter, \& Tomasello, 2005). Of particular interest to the studies presented here, Esteve-Gibert, Prieto, and Liszkowski (2016) showed that 12-month-old infants interpret pointing gestures differently depending on the particular shape of the pointing hand and how the adult vocalized while pointing. When the adult pointed to an object with the index finger and vocalized excitedly (as if wanting to share interest), infants simply attended to the object. When the adult used a palm-up whole hand pointing gesture and vocalized short syllables (as if requesting), infants attended to the object but also offered it to the adult. These results provide good evidence that already very young children integrate different social cues when interpreting others' communicative acts.

Although much more subtle, gaze cues are equivalently powerful in directing even very young children's attention. Beginning in the first year, infants follow gaze to a target in the presence (Senju \& Csibra, 2008; Senju, Csibra \& Johnson, 2008) and absence of ostensive cues (Gredebäck, Astor \& Fawcett, 2018), and even in the absence of a salient congruent head turn (Tomasello, Hare, Lehmann \& Call, 2007). At the end of the second year, children are able to use both pointing as well as gaze with and without head turn to find a hidden toy in an object-choice task (Itakura \& Tanaka, 1998). Taken together, children at the end of the second year are well able to follow both points and gaze cues to retrieve hidden objects in forced-choice paradigms.

Specific challenges arise when directional cues are combined with salient negative emotional displays, that is when children have to avoid an item that is referred to in a negative way by an interlocutor. Already 17-month-olds can avoid choosing an incorrect 
location when it is pointed at, but only if they had visual access to the hiding place of the desired item (Southgate, Chevallier, \& Csibra, 2017). In the absence of directly conflicting evidence, recovering the meaning of a communicative act involving a referential gesture and a negative facial expression in an object choice situation requires some kind of disjunctive reasoning $(\mathrm{P} \vee \mathrm{Q} ; \neg \mathrm{P} \rightarrow \mathrm{Q}$ ). The listener has to reason that the location that is being communicated about is not the location that contains the desired object. Therefore, it must be in the other location instead. Children solve basic versions of disjunctive reasoning tasks from 22 months onwards (Austin, Theakston, Lieven, \& Tomasello 2014; Mody \& Carey, 2016). ${ }^{1}$ In the context of the present studies, 22 -months is the youngest age-group we tested.

However, there is evidence suggesting that even younger children can pragmatically integrate negative displays in an object-choice-scenario. Repacholi (1998) presented 14- and 18-month-old children with an object-choice task in which an actor picked up two boxes in quick succession and emoted positively to the content of one and negatively towards the other. Infants preferred to touch and search the positively indicated box in all conditions. However, the operationalization with two cues limits the interpretation of participants' responses crucially. To solve the task, children would not necessarily have to understand the negative cue but could have simply relied on the positive cue alone. To really test young children's ability to use a negative referential cue, it is necessary to show that they systematically avoid one of two objects when only one of them is referred to negatively.

In fact, previous studies on epistemic vigilance and testimony suggest that young children tend to "blindly" follow referential cues, regardless of their embedding. Children up until the preschool years have a very strong tendency to follow testimony (Jaswal, Croft,

\footnotetext{
${ }^{1}$ Recent evidence suggests that even 12-month-olds can compute logical inferences of this sort (Cesana-Arlotti, Martín, Téglás, Vorobyova, Cetnarski \& Bonatti, 2018), however, a more parsimonious object tracking account might suffice to explain the findings (Jasbi, Bohn, Long, Fourtassi, Barner \& Frank, 2019).
} 
Setua, \& Cole, 2010, Mascaro \& Sperber, 2009) - and pointing gestures in particular (Couillard \& Woodward, 1999; Grassmann \& Tomasello, 2010; Stengelin, Grüneisen \& Tomasello, 2018; Palmquist \& Jaswal, 2012; Palmquist, Kondrad \& Norris, 2018) - even when other information tells them not to. In an object-choice-task, 3-year olds consistently follow the advice of an informant who is introduced as a "big liar". Only four-year-olds were able to overcome this tendency (Mascaro \& Sperber, 2009). Vanderbilt, Liu, and Hayman (2011) found that three- and four-year-olds were equally willing to accept advice from helpers and hinderers who consistently provided correct or incorrect advice respectively (Vanderbilt, Liu \& Heyman, 2011). Three-year-olds continuously followed wrong advice from a speaker claiming that a sticker was in one location when it actually was in another, but they learned to search in the opposite location when the experimenter placed an arrow instead of using verbal testimony (Jaswal, Croft, Setua, \& Cole, 2010). Pointing gestures not only trump other types of reference but are also particularly hard to ignore for young children. Three-year-olds are more likely to hand over an object that an experimenter highlighted via pointing when she looked and pointed at two different objects simultaneously (Lee, Eskrit, Symons \& Muir; 1998), and 2- and 4-year-olds follow pointing over linguistic cues when learning labels (Grassmann \& Tomasello, 2010). In the context of a competitive game, 5- and 7-year-olds are only beginning to spontaneously consider others' incentives when interpreting pointing gestures of a partner who wants them to not find a toy in a search game (Stengelin, Grüneisen \& Tomasello, 2018). However, they stop trusting after having received false information once. The most striking findings are provided by studies on deceptive pointing. Three- and 4-year-olds consistently fail to reject the advice of an overtly misleading informant (Couillard \& Woodward, 1999; Heyman, Sritanyaratana \& Vanderbilt, 2013). 
One reason why children have such a hard time to reject deceptive or misleading advice could be limitations in their executive functioning (Couillard \& Woodward, 1999; Palmquist \& Jaswal, 2011; Palmquist, Kondrad \& Norris, 2018). Overcoming the prepotent response of taking the indicated option is then most likely in cases where responses to cues are highly entrained such as the pointing gesture (Heyman, Sritanyaratana, \& Vanderbilt, 2014; Palmquist, Burns \& Jaswal, 2012; Palmquist \& Jaswal, 2012; Palmquist, Kondrad \& Norris, 2018). Another possible explanation is that children generally assume that acts of intentional communication are truthful (truthful communicator bias). Evidence for this explanation is provided by studies showing that children are better able to reject a cue when its presentation (i.e. a flashing light as an indicator) is dissociated from an interlocutor (study 5, Heyman, Sritanyaratana, \& Vanderbilt, 2014). If the communicative intent, however, was the only factor influencing children's performance they should perform equally well with different types of cues.

In the studies presented here, we seek to extend research on children's ability to integrate emotional and referential cues. Study 1 contrasted the integration of positive and negative facial expressions with pointing gestures in 22-month-olds in a between-subjects-design. Following up on the results of Study 1, Study 2 traced the development of children's ability to integrate negative expressions with pointing gestures in 28-, 34-, 40-, and 46-month-old children. Study 3 investigated the integration of a different kind of referential cue (peeking under the box) with negative facial expressions in 22-, 28-, and 34-month-olds. In contrast to earlier studies in which both emotions and gestures referred to "things in the world", the signals we studied had a proto-syntactic structure. In an object choice task, a pointing gesture was used to single out a referent and the valence of an emotional expression (positive or negative) - which was not directed at the referent - 
determined whether the speaker intended the child to act or refrain from acting on the referent.

The set of studies presented here complements and goes beyond several lines of work investigating children's ability to integrate contextual and multimodal information when making pragmatic inferences. First of all, it investigates a broader age-range than most studies (Couillard \& Woodward, 1999; Heyman, Sritanyaratana \& Vanderbilt, 2013) and follows children's development from the end of the second year to the end of the fourth year in six-months-intervals. Other than previous work requiring children to avoid a negatively indicated option, we do not provide children with a positive and negative cue in the same trial as this would make the pragmatic inference obsolete. To evaluate the special status of the pointing gesture for children's performance, we have replaced pointing with gaze which is ecologically more valid than the use of a marker (Couillard \& Woodward, 1999) or placing an arrow (Jaswal, Croft, Setua, \& Cole, 2010). Furthermore, in many studies using combinations of emotional and directional information it is unclear if children understand emotional expressions as a message in and of itself or simply see it as a reaction towards the target object. In previous studies, the adult either directly touched (Repacholi, 1998) or looked (Moses et al., 2001) at the referent while emoting the emotional expression was probably seen as an involuntarily produced reaction to the stimulus. Children might have exploited the adult's reaction to adjust their own behavior. It is unclear if children can use valence information if the emotional expression is intentionally produced for them. Here we study this by looking at children's ability to integrate referential information provided by a pointing gesture with emotional facial expressions. While pointing can be used to indicate a referent without directly looking at it, an emotional expression produced while looking at a 
recipient, instead of the referent, is an intentional communicative act instead of an involuntary reaction.

\section{Study 1}

\section{Participants}

All children that were tested in the studies reported here came from a medium-sized middle-European city of predominantly Caucasian population. They were found via a database of subjects for child development studies to which their parents had voluntarily signed up. Appointments were made on the basis of parents' and children's availability. All studies described below were reviewed and approved by an internal ethics committee at the [blinded].

We invited 20 children in each condition and age-group. In Study 1, twenty 22-month-olds participated in the positive pointing condition (mean age $=22.03$ months, SD $=1.12$ months, range $=20.32-23.83$ months; 10 boys). Another group of twenty 22-month-olds was tested in the negative pointing condition (mean age $=22.50$ months, $\mathrm{SD}=$ 1.08 months, range $=20.51-23.80$ months; 10 boys). Additionally, six further children (positive condition $n=2$; negative condition $n=4$ ) were tested but not included in the analysis due to parental interference $(n=1)$ and because they were fussy and did not complete the procedure $(n=5)$. Sample size for each group was pre-planned and based on earlier studies using a similar setup (Behne, Carpenter, \& Tomasello, 2005).

\section{Procedure and setup}

Upon arrival in the laboratory, children were invited to play with a female experimenter ( $\mathrm{E}$ - same person in all studies) in a play room for 15-20 minutes until the 
children felt comfortable giving and taking toys with her. During this phase, parents filled in consent forms and were instructed not to influence the child's behavior at test.

Next, children and parents were led into the testing room and children were invited to play a hiding game that set the context for the test trials. E1 first presented them with a marble and a small piece of tissue. Children learned that the marbles could be fed into a papier-mâché elephant to make a chime play. The piece of tissue had no relevance in the context of the game. Next, E would present two identical small boxes serving as hiding places. E presented the marble and the piece of cloth, put them side by side and covered them with the boxes. The child received the prompt: "Where is the marble?" and could then retrieve the marble from under the box to feed the toy elephant. The hiding game was repeated for three trials in total. The warm-up procedure was designed to ensure that children knew that the marble would be hidden under one of the two boxes whereas the other one would be baited with the tissue. Furthermore, the three trials showed that children felt comfortable in lifting the boxes, were motivated to search the marble and preferred to find the marble over to the piece of tissue.

For the actual test set-up, children were invited to sit or stand facing the experimenter at approximately $120 \mathrm{~cm}$ distance. A small table in front of the experimenter served as a tray for the hiding game. The table was approximately $120 \mathrm{~cm}$ wide and $30 \mathrm{~cm}$ high. The boxes for the hiding game were placed in the corners of the table such that children could reach them conveniently upon walking over, but not lift both boxes at once when making a choice. A visual occluder of $100 \mathrm{~cm} \times 80 \mathrm{~cm}$ was placed in front of the table to block visual access to the table during the baiting of the boxes. The soft felt surface of the table ensured that children could not hear where the marble was placed. To keep children alert and mark the 
beginning of a new trial, E used a new pair of identical boxes in different colors for every trial. For an illustration of the set-up, please see Figure 1.

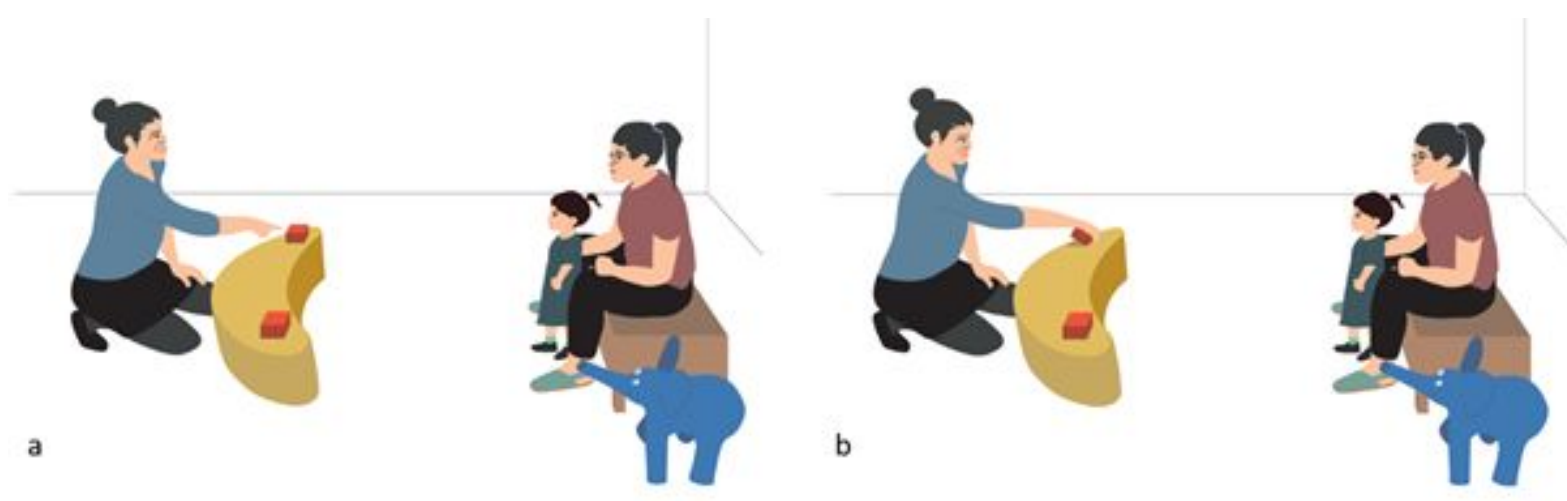

Figure $1 a \& b$. Schematic drawing of the setup. The left panel (a) shows how the experimenter indicated a location by pointing in Study 1 and 2, the right panel (b) shows indicating by peeking in Study 3. Children made a choice by approaching and touching one of the boxes.

In each test trial, E placed a pair of boxes for the hiding game on the table. Boxes were upside down such that the child could see that they are empty. E then placed a piece of tissue in the middle of the table, called the child's attention and presented a marble. Once the child was attentive, E placed the occluder in front of the table to block visual access to the hiding event. E continued by taking the marble and tissue in both hands in the middle of the table. Next, she covered each item with a box and then moved both boxes simultaneously to the opposite ends of the table.

For the pointing event, E removed the occluder and called the child's name to get her attention. Depending on the condition, E first made a positive or negative facial expression while looking at the child. Then she returned to a neutral facial expression and both pointed and looked at one of the boxes. Next, E retained the point, looked at the child again and repeated the emotional expression. In case the child was inattentive, the pointing procedure 
was repeated such that the child had seen the emotional expression at least twice in each trial. Throughout the trial, the emotional expression was distinctly and only displayed while E was looking at the child. This is important as the emotional expression was not presented as an immediate reaction to the target itself but rather meant to serve a communicative function.

The decision phase was initiated by E prompting the child to make a choice saying "Where is the marble?". Then, the child could walk to the table to open one of the two boxes. If the child found the marble, she could feed the elephant. In case she lifted the box with the tissue, E turned over the box with the marble and handed it to the child. If the child did not make a choice, E repeated the pointing event once. If the child still didn't make a choice, E ended the trial by lifting both boxes and also handing the marble to the child. Children could feed the elephant in each case. Rewarding participants non-differentially kept them engaged and counteracted demand effects that could have resulted from the games' pay-off structure.

In Study 1, we employed two experimental conditions: a positive condition and a negative condition. In the positive condition, E showed a positive (happy, friendly and interested) expression on her face, accompanied by a matching vocalization (“Ahh”), and pointed to the box where the marble was hidden. This condition corresponded to a standard object choice task. In the negative condition, E displayed a negative emotional expression (aversive, disgusted and suspicious) before pointing, again accompanied by a matching vocalization ("Eww"). In this condition, E always pointed to the box with the tissue (i.e. the irrelevant box). To find the marble and succeed in the hiding game, children had to avoid the box that E was pointing at.

Rather than displaying specific emotional expressions such as anger or disgust in the negative condition or surprise in the positive condition, E used an amalgam of positive and negative emotional expressions respectively. This made the display highly salient. The 
positive facial expression involved Action Units (AU) that are generally seen as pleasant (Scherer \& Ellgring, 2007): AU1 (Inner Brow Raiser) and AU2 (Outer Brow Raiser), AU 12 (Lip Corner Puller) together with AU 25 (Lips Part), and AU26 (Jaw Drop, Open Mouth), indicating a happily surprised face. The negative expression was disgust-like and involved AUs that are related to unpleasantness (Scherer \& Ellgring, 2007): AU 4 (Brow Lowerer), AU 6 (Cheek Raiser), AU 7 (Lid Tightener), AU 9 (Nose Wrinkler), AU 10 (Upper Lip Raiser), AU 16 (Lower Lip Depressor) and also AU 25 (Lips Part). For photographs of the facial expressions, see Figure 2.
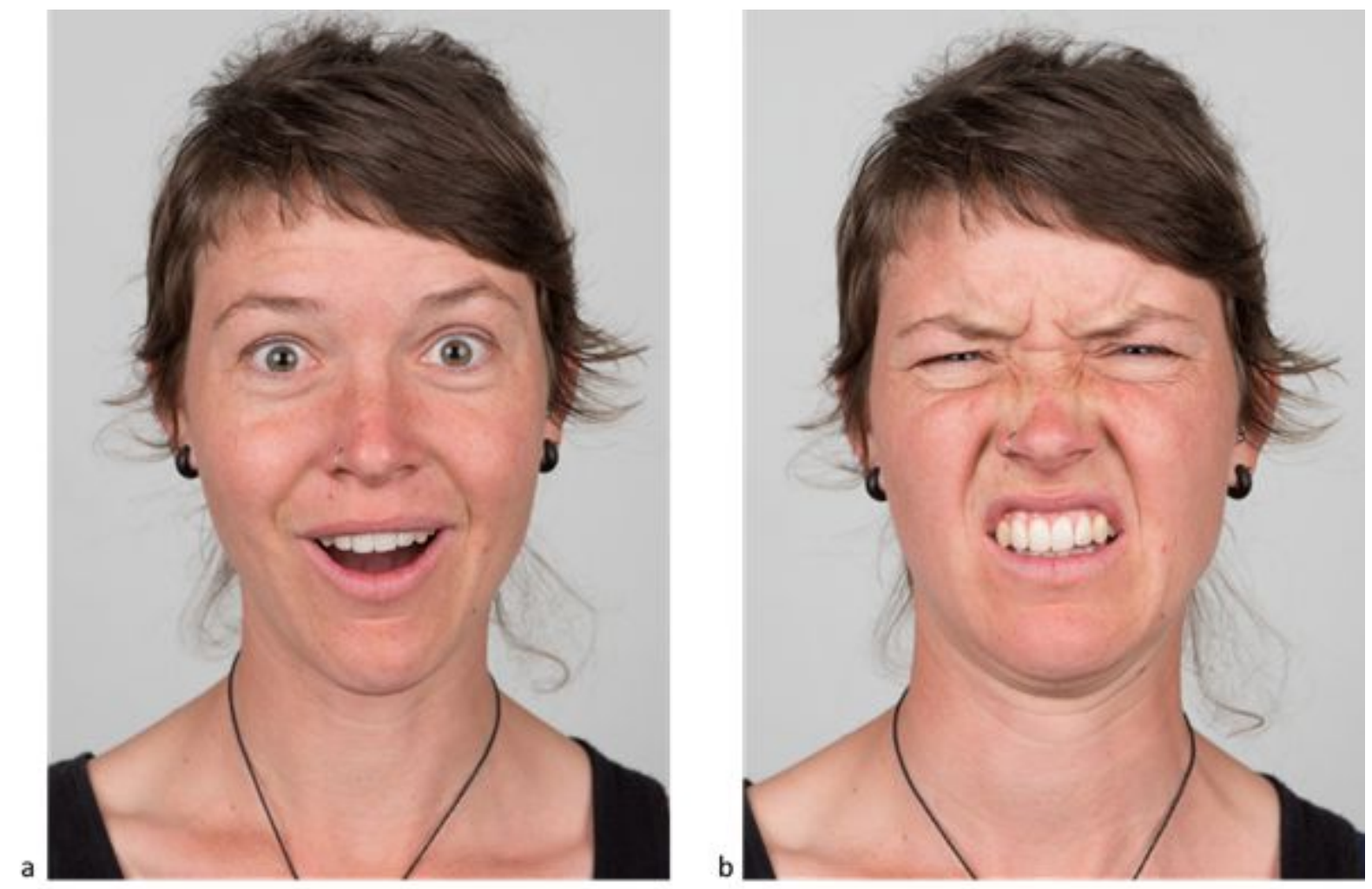

Figure $2 a \& b$. Illustration of the positive (a) and negative (b) facial expressions made by the experimenter. The experimenter was the same person in all studies. Detailed FACS coding of these expressions can be found in the main text. 
Notably, whereas the experimenter in the procedure employed by Repacholi (1998) provided a positive and negative cue in each trial, the experimenter in our set-up always pointed to one of the two boxes only. In a between-subjects design, children were either tested in the positive or negative condition. Hence, each child only saw E either point positively to the location containing the marble or point negatively to the box baited with the piece of tissue. The direction of the point and the location of the two items were pseudo-randomized with E never pointing to the same box more than twice in a row. Children were tested for eight consecutive trials.

\section{Results and discussion}

In all studies, we coded as choice the container that the child touched first (left or right from the experimenter) and whether this was the correct container (i.e. with the marble). For each group in each study, a second coder re-coded $25 \%$ of trials. Lower bound of agreement between coders across studies was $92.5 \%(\kappa=0.85)$.

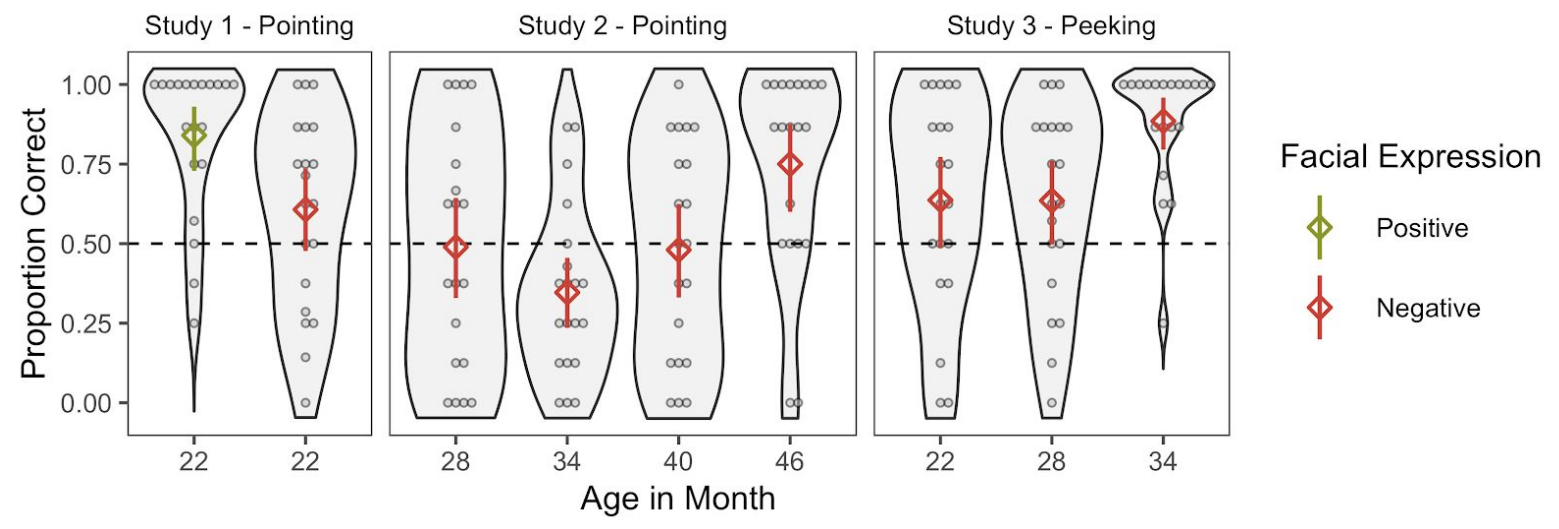

Figure 3. Proportion of correct response per age group and study. Diamonds show group means, error bars represent $95 \%$ confidence intervals around the mean based on non-parametric bootstraps. The dotted line indicates performance expected by chance. Black circles show individual data with the number of the circles corresponding to the number of participants with that value. Smoothed density distributions are shown in grey. 
Table 1

Summary statistics for performance compared to chance (.5) within each group.

Individuals

performing

\begin{tabular}{|c|c|c|c|c|c|c|c|c|c|c|}
\hline Study & Age & Cue & $\mathrm{FE}$ & M & $\mathrm{SD}$ & $t(19)$ & $p$ & Cohen's $d$ & $<.5$ & $>.5$ \\
\hline \multirow[t]{2}{*}{1} & 22 & Point & positive & 0.84 & 0.24 & 6.46 & $<.001$ & 1.45 & 0 & 11 \\
\hline & 22 & & negative & 0.61 & 0.31 & 1.57 & .133 & 0.35 & 1 & 3 \\
\hline \multirow[t]{4}{*}{2} & 28 & Point & negative & 0.49 & 0.37 & -0.12 & .902 & 0.03 & 4 & 4 \\
\hline & 34 & & & 0.35 & 0.27 & -2.55 & .020 & 0.57 & 3 & 0 \\
\hline & 40 & & & 0.48 & 0.34 & -0.26 & .800 & 0.06 & 3 & 1 \\
\hline & 46 & & & 0.75 & 0.32 & 3.47 & .003 & 0.78 & 2 & 8 \\
\hline \multirow[t]{3}{*}{3} & 22 & Peek & negative & 0.64 & 0.33 & 1.83 & .083 & 0.41 & 2 & 5 \\
\hline & 28 & & & 0.63 & 0.31 & 1.97 & .064 & 0.44 & 1 & 3 \\
\hline & 34 & & & 0.88 & 0.20 & 8.80 & $<.001$ & 1.97 & 0 & 12 \\
\hline
\end{tabular}

Note. $\mathrm{FE}=$ Facial expression. Evaluation of group level performance is based on two-tailed one samplet-tests. Individual performance is evaluated through two-tailed binomial tests with none or all trials correct resulting in $\mathrm{p}<.05$.

Table 1 and Figure 3 give an overview of the performance within each group compared to a performance expected by chance. On a group level, performance did not differ from chance in the negative condition but did so in the positive condition. A closer look at the distribution of the data in Figure 3 shows that participants varied substantially in their performance in the negative condition. Some children performed above chance on an 
individual level, suggesting that success in the task is not entirely beyond children's ability at this age (see Table 1).

To directly compare performance in the two conditions, we fit a generalized linear mixed model to the trial by trial data with condition and trial as predictors. All models were fit in r (R Core Team, 2017) using the package lme4 (Bates, Mächler, Bolker, \& Walker, 2015). All models included random intercepts for participant and random slopes for trial. P-values for predictors are based on likelihood ratio tests obtained through single term deletions (Dobson \& Barnett, 2008). Data and analysis code associated with the study can be found in the following online repository:https://github.com/manuelbohn/emopoint.git

Children performed better in the positive compared to the negative condition $(\beta=$ $-1.90, \mathrm{se}=0.66, \mathrm{p}=.003)$. Performance further tended to increase in later trials $(\beta=0.39$, se $=0.21, \mathrm{p}=.050)$. This pattern suggests that children paid attention to the emotional facial expression of the experimenter and integrated it with the pointing gesture. If children had ignored the facial expression altogether and only followed the point, performance in the negative condition should have been below chance. While this might have been the case for some individuals, it was not the dominant behavior in the group. Nevertheless, children performed much better when the emotional expression encouraged them to approach the indicated location. To succeed in the negative condition, children had to inhibit approaching the indicated location, evaluate the facial expression. That is, children had to exclude the alternative that E pointed to and approach the other one instead.

Previous studies on children's referential understanding of emotions (e.g. Repacholi, 1998) presented positive and negative emotions together, thereby omitting the need for excluding one alternative. However, based on these results alone, it remains unclear, at what age children (on a group level) can infer the intentions behind referential communicative acts 
including negative emotions. In Study 2, we therefore followed the developmental trajectory of children's performance in the negative condition. We expected a linear increase in children's performance with age. We tested additional age groups in six-month intervals until we found group level performance to be above chance level.

\section{Study 2}

\section{Participants}

In Study 2, we tested four age-groups with twenty children. Following up on Study 1, we started with twenty 28 -months-olds (mean age $=28.86$ months, $\mathrm{SD}=0.99$ months, range $=26.3-29.91$ months; 10 boys) and progressed in intervals of six months to the next age-groups: 34 -month-olds (mean age $=34.88$ months, $\mathrm{SD}=0.63$ months, range $=33.69-$ 35.80 months; 10 boys) and 40-month-olds (mean age $=40.19$ months, $\mathrm{SD}=1.11$ months, range $=38.17-41.98$ months; 10 boys). The oldest age-group were 46-month-olds (mean age $=46.90$ months, $\mathrm{SD}=0.88$ months, range $=44.41-47.96$ months; 10 boys). One additional 28-month-old child was tested but excluded from the analysis due to fuzziness.

\section{Procedure and setup}

The procedure and setup were identical to the negative condition in Study 1 .

\section{Results and discussion}

Group level summary statistics for Study 2 can be found in Table 1 and Figure 3 . The distribution of performance in each age group again showed great variability between participants. On a group level, performance was below chance at 34 months (with no child performing above chance) and above chance at 46 months. In contrast to our expectation, performance did not increase in a linear fashion with age. Visual inspection of Figure 3 suggested a u-shaped development instead. We confirmed this visual impression statistically 
by fitting a model to all data from the negative condition. This model included all data from Study 2 and the negative condition from Study 1. For this analysis, we treated age as a continuous variable across groups. There was no linear effect for age $(\beta=0.27, \mathrm{se}=0.23, \mathrm{p}=$ .235). Adding a quadratic term for age to this model significantly improved the fit $\left(\chi^{2}(1)=\right.$ 14.98, $\mathrm{p}<.001)$. The estimate for the quadratic term was positive $(\beta=1.00$, se $=0.25)$, confirming the convex shape of the trajectory. Furthermore, performance increased in later trials $(\beta=0.25, \mathrm{se}=0.09, \mathrm{p}=.007)$. Fitting a model with age as categorical predictor and 34 months as reference group showed that performance at 34 months was worse compared to 46 months $(\beta=1.76, \mathrm{se}=0.66, \mathrm{p}=.008)$ and also trended to be below performance at 22 months $(\beta=1.08, \mathrm{se}=0.59, \mathrm{p}=.069)$.

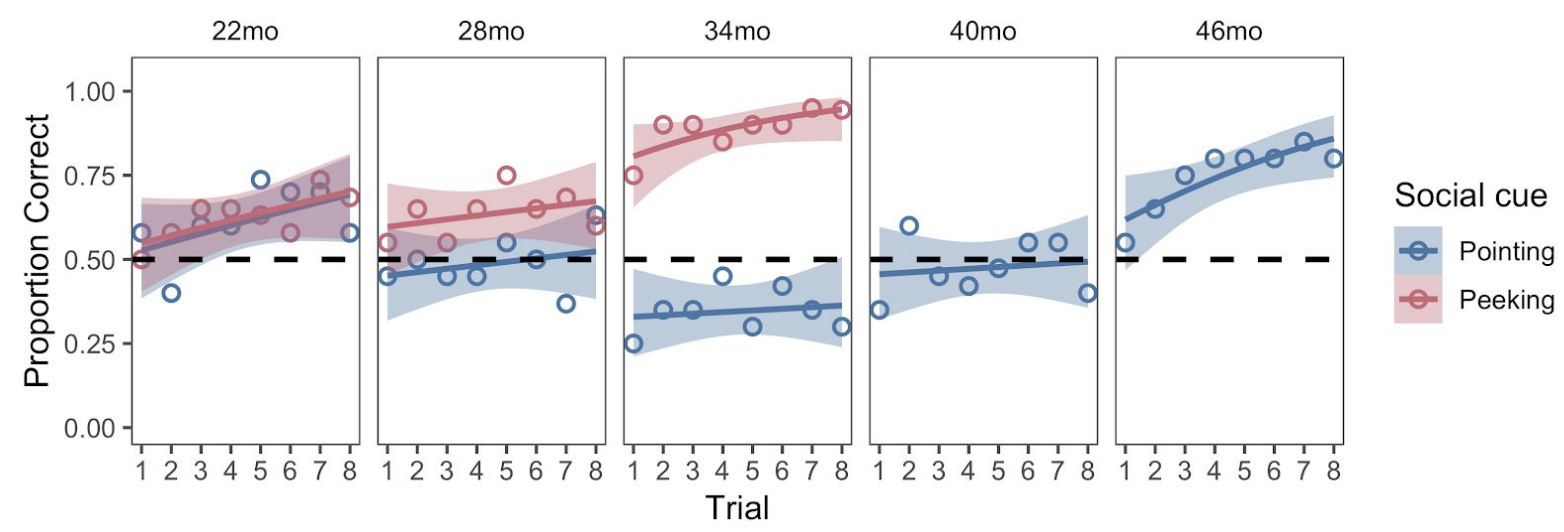

Figure 4. Proportion correct choice by age group and trial for conditions with negative facial expressions. Regression lines show smoothed conditional means with 95\% confidence intervals. Forty- and 46-month-olds were not tested in peeking because performance was above chance at 34 months.

The pattern of results observed in the negative conditions suggests that children (at a group level) do not succeed in integrating negative emotional expressions with pointing gestures until 46 months of age. Furthermore, the tendency to act upon the indicated object or location becomes stronger, apparently leading 36-month-olds to ignore the facial expression 
altogether. Figure 4 shows that performance in this age group remained below chance across all trials, which suggests that the behavior is relatively robust against experience.

One explanation for the U-shaped development is that pointing gestures do more than indexing a referent. They also create a tendency to act upon the pointed to location/object, suggesting an affirmative connotation. This "secondary meaning" of pointing gestures is less pronounced in younger children and increases with experience (approximated by age in our study), leading older children to ignore additional cues to a speaker's intention when the gesture is used in a way that differs from the usual way. From 34 months onwards, children get better at inhibiting their initial response tendency, making room for integration with the facial expression. This interpretation is supported by the trial by trial performance of the 46-month-olds. Performance in trial 1 is around chance with a steep increase in the following trials, suggesting an initial tendency (at least in some children) to ignore the facial expression and follow the pointing gesture.

Alternatively, children might not struggle with the integration of cues but with inferring that the negatively indicated location does not contain the desired object, which could also lead to a u-shaped developmental trajectory. In a third study, we therefore investigated the development of children's inferential abilities in a similar task, reducing the need to integrate. Instead of pointing to the container and subsequently emoting towards the child, the experimenter peeked under the container and displayed the emotional expression while looking at its content.

\section{Study 3}

\section{Participants}

In Study 3, we tested children of three ages with twenty participants in each group. To complement Study 1, we started with 22-month-olds (mean age $=23.35$ months, $\mathrm{SD}=0.48$ 
months, range $=22.35-24.00$ months; 9 boys) and then went on to test 28 -month-olds (mean age $=28.59$ months, $\mathrm{SD}=1.04$ months, range $=26.36-29.75$ months; 10 boys $)$ and 34-month-olds (mean age $=35.35$ months, $\mathrm{SD}=0.45$ months, range $=34.48-35.93$ months; 11 boys). Additionally, five further children were tested but not included in the analysis (22-month-olds: $\mathrm{n}=3 ; 34$-month-olds; $\mathrm{n}=2$ ). These children were excluded due to restlessness $(n=3)$ or because they were too shy to make a choice $(n=2)$.

\section{Procedure and setup}

The procedure and set-up for Study 3 were identical to those described for Study 1 and 2. However, the pointing gesture was replaced with an act of peeking under the boxes. In the test trials of Study 3, E removed the occluder and called the child's name. Then, E looked at the incorrect box (i.e. the one baited with tissue), reached for it and lifted it up slightly such that she could see its content but the child could not. Upon lifting the box, E started to make a negative facial expression (see Figure 2). Then, E looked back at the child and removed her hands from the box. In contrast to the pointing events in Study 1 and 2, the emotional expression was displayed only once and while looking at the target. However, E's behavior was still framed as a communicative act rather than a reaction towards the content of the box due to the attention getter and the pragmatics of the hiding game. If the child was inattentive during the peeking event or did not make a choice, the peeking event was repeated.

\section{Results and discussion}

Table 1 and Figure 3 summarize performance per group in Study 3. Performance tended to be above chance already in the two younger age groups, again with substantial variability across participants and multiple individuals performing above chance in each 
group. At 34 months of age, the task appeared to be almost trivial for the children, with more than half of the group being $100 \%$ correct (see Table 1). To evaluate the developmental trajectory, we fit a model with a linear predictor for age. This turned out to be positive and significant $(\beta=0.65$, se $=0.25, \mathrm{p}=.009)$, corroborating the absence of a u-shaped trajectory. Performance further increased with trial $(\beta=0.78$, se $=0.26, \mathrm{p}>.001)$. A change in inferential abilities as outlined above is, therefore, unlikely to explain the trajectory in Study 2.

Comparing the results from Study 2 and 3 showed a separation of inferential and integrative abilities in the task at hand. At 34 months, children selected the correct container below chance when the negative facial expression had to be integrated with a preceding point but their performance was above chance when the same expression was displayed while looking at the target. In both cases, children had to exclude the indicated container in favor of the alternative. No such pattern should have been observed if children would generally struggle with avoiding the highlighted container. Rather it seems that pointing gestures, presumably due to how they are used in everyday life, do more than just indicate objects for children; they have an additional "secondary meaning", encouraging children to approach or act upon the indicated object.

\section{General discussion}

In this set of studies, we investigated the development of children's ability to integrate facial expressions and pointing gestures. Study 1 found that 22-month-olds are better at recovering the speaker's intended meaning when the pointing gesture is accompanied by a positive as opposed to a negative facial expression. Study 2 followed the developmental trajectory of the ability to integrate negative expressions and pointing in 28-, 34-, 40-, and 46-month-olds, finding a u-shaped developmental pattern. Study 3 showed that the u-shaped pattern was not 
due to difficulties in syllogistic reasoning in this context. These results suggest that, early in development, pointing gestures carry an affirmative meaning and do more than just indicate a referent.

The results from Study 1 mirror findings from work using verbal prompts (Grigoroglou, Chan, \& Ganea, 2019), in which younger two-year-olds performed better with affirmative as opposed negative sentences in search task. In contrast to (Grigoroglou, Chan, \& Ganea, 2019), we did not find that performance increased steadily with negative prompts over the second year. Rather, the results of Study 2 were unexpected. Although children seem to be able to integrate referential cues and negative emotional cues at 12 months (Moses, et al., 2001), 22- to 40-month-olds struggled to integrate a pointing gesture and a negative facial expression. Performance even decreased between 22 to 34 months of age. How can we explain this u-shaped developmental trajectory? We suspect that two staggered developmental processes are at work here. On the one hand, children experience communicative acts in general and pointing gestures in particular to be used mainly in veridical and affirmative ways, that is, to provide true information or to encourage engagement with a referent (Heyman, Sritanyaratana, \& Vanderbilt, 2014; Jaswal et al., 2014; Palmquist, Burns \& Jaswal, 2012; Palmquist \& Jaswal, 2012). Following Karmiloff-Smith (1992), children might develop a procedural understanding of pointing in that seeing a point to an object triggers the dominant response of approaching or acting upon the pointed to object. The purely indexical function of pointing would therefore not be immediately accessible, which would prevent children from integrating additional contextual information. In line with our results in Study 2, a number of studies suggest that this process culminates at three years of age. For example, children of that age follow a clearly ignorant pointer's gesture, even if it directly conflicts with a reliable gesture from a knowledgeable 
communicator (Palmquist \& Jaswal, 2012, Palmquist, Burns \& Jaswal, 2012). Furthermore, three-year-olds struggle to ignore pointing gestures to one of two containers even if these points are constantly misleading in several consecutive trials (Couillard \& Woodward, 1999). This response seems to be less pronounced in younger children, as for example two-year-olds differentiate between points from an adult compared to points from a peer (Kachel, Moore \& Tomasello, 2018). However, at four years of age, children are able to avoid following pointing gestures in an object-choice-task when an experimenter explicitly states that she is going to point where the target item is not hidden (Palmquist, Kondrad \& Norris, 2018).

The results of Study 3 suggest that the dominant response in three-year-olds is specific to pointing and does not generalize to an equivalent directional cue, namely E's gaze. When the experimenter peeked under the box, thereby highlighting it spatially, three-year-olds considered the emotional expression and refrained from approaching the box. This is in line with work by Mascaro and Sperber (2019), who found that three- to four-year-olds were able to learn the rule that a marker (study 1) or arrow (study 2) marked the location of an empty container in an object-choice task with a cooperative (i.e. non-deceptive) partner. They, too, conclude that reinterpreting a well-established signal such as pointing is particularly difficult for young children (Mascaro and Sperber, 2019).

The development of a procedural understanding of pointing might explain the decline in performance in our study throughout the second year. On the other hand, children's executive functions in general and inhibitory skills in particular improve substantially in the fourth year of life. In tasks asking participants to inhibit a predominant response, such as the day-night task, four-year-olds generally outperform three-year-olds (see e.g. Carlson \& Moses, 2001; Garon, Bryson \& Smith, 2008; Müller, et al., 2012). In our studies, the improvement in performance from 34- to 46-month of age might reflect the increasing ability 
to inhibit the prepotent response and consider additional contextual information. This could go along with a process of children representationally re-describing pointing as a purely indexical communicative act on a more explicit level (Karmiloff-Smith, 1992). Explicit, as opposed to procedural, representations allow for an integration of information from different sources. Initially, children might still be tempted to follow the pointing gesture as 46-month-olds' performance is at chance in trial 1 (see Figure 4). However, performance increases rapidly in subsequent trials, showing that children are able to eventually overcome this prepotent tendency. This hypothesis could be tested by correlating performance in our task with other measures of inhibitory skills. Following Yurovsky and Frank (2017), we may conclude, for now, that the integration of different social cues depends on a gradual development of domain general abilities.

Comparing children's performance with true negative points and overtly deceptive points, Palmquist, Kondrad and Norris (2018) found performance above chance for true negative points at four years of age which is perfectly in line with our findings. The authors interpret their finding to indicate that children's inability to neglect information from deceptive pointing (Heyman et al., 2013; Couillard \& Woodward, 1999) stems from their expectation that pointers are truthful (truthful-pointer bias) rather than from their expectation about the meaning of the gesture itself (point bias, cf. Palmquist, Kondrad and Norris; 2018). Our studies can further qualify this finding by showing that four-year-olds even succeed in a non-verbal version of the same task, and by showing that even younger children perform above chance when pointing is replaced by ostensively looking at the item to be avoided. We may conclude that while even two-year-olds are generally able to avoid negatively referenced targets, they still struggle with overcoming a pointing bias at three, but go on to overcome a truthful pointer bias only after the age of four. 
The interpretation offered here focused on children's mean performance in each age group. It is, however, worthwhile to look at the distribution of the data in each condition. In general, there is a large amount of variability in each group and in all conditions. Except in the negative pointing condition at 34 months of age, some children perform above chance on an individual level (see also Table 1). This suggests that successful integration of negative facial expressions and pointing gestures is already possible at an earlier age. Palmquist, Kondrad and Norris (2018) combined their task with different tests for children's inhibitory control, they found that individual performance was correlated with higher-order inhibitory control as measured by a dimensional change card sort task but not with children's performance in a simpler Grass-Snow task (Palmquist, Kondrad \& Norris, 2018). The finding underscores that not searching a location that was pointed at requires children to inhibit the prepotent response of following points.

To the extent that a correct response in our task requires the inversion of a positive message by combining it with a negative emotional cue, it has similar demands as tasks employed to investigate the onset of irony comprehension. In this line of work, the meaning of a positive utterance is usually inverted by a special tone of voice (Hancock, Dunham, \& Purdy, 2000, Wilson, 2013; Dews et al. 1996) requiring the parallel processing of two communicative cues by the recipient (Pexman, 2008). Children are generally described to separate literal from intended speaker meaning only around five to six years of age (Hancock, Dunham, \& Purdy, 2000). Given that children succeed in our set-up at 46 months in the pointing and at 34 months in the peek condition, our work might inform this literature by highlighting that the late onset of irony comprehension is unlikely to be due to children's problems with cue integration per se (cf. Wilson, 2013). 
Finally, our results highlight that children up into the preschool years are prone to understand reference expressed via pointing gestures as a call to act upon an object regardless of a saliently negative emotional embedding. This has important consequences for pedagogical interactions on a day-to-day basis. Children's ability to integrate an act of reference with a negation or behavioral restriction is critical for their understanding of prohibitory and safety rules (“Don't touch that!”; "Please, don't go there!”, "You can't eat this!"). Even toddlers encounter such restrictive directions regularly in everyday life (Dunn \& Munn, 1987; Kopp, 1982; Smetana, Kochanska, \& Chuang, 2000) making them a crucial context for the mastering of negation in general (Choi, 1988; Pea, 1980). However, any prohibitive statement also highlights its referent since children's attention is drawn to objects that are negated in linguistic statements (Nordmeyer \& Frank, 2014), and pointing gestures are construed as affirmative, which makes it very hard for children to comply with an adults' directions and inhibit their tendency to further approach what has been prohibited. Thus, safeguarding children effectively may require safety rules and behavioral constraints to be made redundantly explicit by combining referential acts with both emotional and linguistic cues, and providing a rich contextualization through explanations and rephrasing of the intended meaning - up until the fourth year of life.

Taken together, our studies show that children enter communicative interactions with certain expectations about speakers. Based on their everyday experience, children expect others' communicative acts to be veridical and affirmative. In many cases, this is a useful strategy in that it provides a rapid interpretation to otherwise ambiguous communicative acts (Bohn \& Köymen, 2018). In some cases, however, it keeps children from considering all the available information, which has important pedagogical implications. With the development 
of executive skills, children are able to eventually overcome their prepotent responses and reliably integrate different sources of information.

\section{References}

Aureli, T., Perucchini, P., \& Genco, M. (2009). Children's understanding of communicative intentions in the middle of the second year of life. Cognitive Development, 24, 1-12. Doi: $10.1016 / j . c o g d e v .2008 .07 .003$

Austin, K., Theakston, A., Lieven, E., \& Tomasello, M. (2014). Young children's understanding of denial. Developmental Psychology, 50(8), 2061. Doi: $10.1037 / \mathrm{a} 0037179$

Bates, D., Machler, M., Bolker, B. M., \& Walker, S. C. (2015). Fitting linear mixed-effects models using lme4. Journal of Statistical Software, 67(1), 1-48. Doi: 10.18637/jss.v067.i01

Behne, T., Carpenter, M., \& Tomasello, M. (2005). One-year-olds comprehend the communicative intentions behind gestures in a hiding game. Developmental Science, 8(6), 492-499. Doi: 10.1111/j.1467-7687.2005.00440.x

Behne, T., Liszkowski, U., Carpenter, M., \& Tomasello, M. (2012). Twelve-month-olds' comprehension and production of pointing. British Journal of Developmental Psychology, 30, 359-375. Doi: 10.1111/j.2044-835X.2011.02043.x 
Bohn, M., \& Köymen, B. (2018). Common ground and development. Child Development Perspectives, 12(2), 104-108. Doi: 10.1111/cdep.12269

Carlson, S. M. and Moses, L. J. (2001), Individual differences in inhibitory control and children's Theory of Mind. Child Development, 72(4), 1032-1053. Doi:10.1111/1467-8624.00333

Cesana-Arlotti, N., Martín, A., Téglás, E., Vorobyova, L., Cetnarski, R., \& Bonatti, L. L.(2018). Precursors of logical reasoning in preverbal human infants. Science, 359(6381),1263-1266. Doi: 10.1126/science.aao3539

Choi, S. (1988). The semantic development of negation: A cross-linguistic longitudinal study. Journal of Child Language, 15(3), 517-531. Doi:10.1017/S030500090001254X

Couillard, N. L., \& Woodward, A. L. (1999). Children's comprehension of deceptive points. British Journal of Developmental Psychology, 17(4), 515-521. Doi: $10.1348 / 026151099165447$

Colonnesi, C., Stams, G. J. J., Koster, I., \& Noom, M. J. (2010). The relation between pointing and language development: A meta-analysis. Developmental Review, 30(4), 352-366. Doi: 10.1016/j.dr.2010.10.001 
Dews, S., Winner, E., Kaplan, J., Rosenblatt, E., Hunt, M., Lim, K., ... \& Smarsh, B. (1996). Children's understanding of the meaning and functions of verbal irony. Child Development, 67(6), 3071-3085. Doi: 10.1111/j.1467-8624.1996.tb01903.x

Dobson, A. J., \& Barnett, A. G. (2008). An introduction to generalized linear models (3rd ed.). Boca Raton, FL: CRC Press.

Dunn, J., \& Munn, P. (1987). Development of justification in disputes with mother and sibling. Developmental Psychology, 23(6), 791. Doi: 10.1037/0012-1649.23.6.791

Esteve-Gibert, N., Prieto, P., \& Liszkowski, U. (2017). Twelve-month-olds understand social intentions based on prosody and gesture shape. Infancy, 22(1), 108-129. Doi: 10.1111/infa.12146

Feiring, C., Lewis, M., \& Starr, M. D. (1984). Indirect effects and infants' reaction to strangers. Developmental Psychology, 20(3), 485. Doi: 10.1037/0012-1649.20.3.485

Flom, R., \& Bahrick, L. E. (2007). The development of infant discrimination of affect in multimodal and unimodal stimulation: The role of intersensory redundancy. Developmental Psychology, 43, 238-252. Doi: 10.1037/0012-1649.43.1.238

Garon, N., Bryson, S. E., \& Smith, I. M. (2008). Executive function in preschoolers: A review using an integrative framework. Psychological Bulletin, 134(1), 31. Doi: $10.1037 / 0033-2909.134 .1 .31$ 
Grassmann, S., \& Tomasello, M. (2010). Young children follow pointing over words in interpreting acts of reference. Developmental Science, 13(1), 252-263. Doi: 10.1111/j.1467-7687.2009.00871.x

Gredebäck, G., Melinder, A., \& Daum, M. M. (2010). The neural basis and development of pointing comprehension. Social Neuroscience, 5, 441- 450. Doi:10.1080/17470910903523327

Gredebäck, G., Astor, K., \& Fawcett, C. (2018). Gaze following is not dependent on ostensive cues: a critical test of natural pedagogy. Child Development, 89(6), 2091-2098. Doi: 10.1111/cdev.13026

Grigoroglou, M., Chan, S., \& Ganea, P. A. (2019). Toddlers' understanding and use of verbal negation in inferential reasoning search tasks. Journal of experimental child psychology, 183, 222-241. Doi: 10.1016/j.jecp.2019.02.004

Hancock, J., Dunham. P. \& Purdy, K. (2000). Children's comprehension of critical and complementary forms of verbal irony. Journal of Cognition and Development, 1:2, 227-248. Doi: 10.1207/S15327647JCD010204

Heyman, G. D., Sritanyaratana, L., \& Vanderbilt, K. E. (2013). Young children's trust in overtly misleading advice. Cognitive Science, 37(4), 646-667. Doi: 10.1111/cogs. 12020 
Itakura, S., \& Tanaka, M. (1998). Use of experimenter-given cues during object-choice tasks by chimpanzees (Pan troglodytes), an orangutan (Pongo pygmaeus), and human infants (Homo sapiens). Journal of Comparative Psychology, 112(2), 119. Doi: 10.1037/0735-7036.112.2.119

Jasbi, M., Bohn, M., Long, B., Fourtassi, A., Barner, D., \& Frank, M. C. (2019). Comment on Cesana-Arlotti et al. (2018). https://doi.org/10.31234/osf.io/g2h7m

Jaswal, V. K., Croft, A. C., Setia, A. R., \& Cole, C. A. (2010). Young children have a specific, highly robust bias to trust testimony. Psychological Science, 21(10), 1541-1547. Doi: $10.1177 / 0956797610383438$

Jaswal, V. K., Pérez-Edgar, K., Kondrad, R. L., Palmquist, C. M., Cole, C. A., \& Cole, C. E. (2014). Can't stop believing: Inhibitory control and resistance to misleading testimony. Developmental Science, 17(6), 965-976. Doi: 10.1111/desc.12187

Kachel, G., Moore, R., \& Tomasello, M. (2018). Two-year-olds use adults' but not peers' points. Developmental Science. Doi: 10.1111/desc.12660

Karmiloff-Smith, A. (1992). Beyond modularity: A developmental perspective on cognitive science. MIT press. 
Klinnert, M. D. (1984). The regulation of infant behavior by maternal facial expression. Infant Behavior and Development, 7(4), 447-465. Doi: $10.1016 / \mathrm{S} 0163-6383(84) 80005-3$

Kopp, C. B. (1982). Antecedents of self-regulation: A developmental perspective. Developmental Psychology, 18(2), 199. Doi: 10.1037/0012-1649.18.2.199

Mascaro, O., \& Sperber, D. (2019). The pragmatic role of trust in young children's interpretation of unfamiliar signals. PloS one, 14(10). Doi: 10.1371/JOURNAL.PONE.0224648

Mascaro, O., \& Sperber, D. (2009). The moral, epistemic, and mindreading components of children's vigilance towards deception. Cognition, 112(3), 367-380. Doi: 10.1016/j.cognition.2009.05.012

Matthews, D., Behne, T., Lieven, E. V. M., \& Tomasello, M. (2012). Origins of the human pointing gesture: A training study. Developmental Science, 15(6), 817-829. Doi: $10.1111 / \mathrm{j} .1467-7687.2012 .01181 . x$

Mody, S., \& Carey, S. (2016). The emergence of reasoning by the disjunctive syllogism in early childhood. Cognition, 154, 40-48. Doi: 10.1016/j.cognition.2016.05.012 
Montague, D. P., \& Walker-Andrews, A. S. (2001). Peekaboo: A new look at infants' perception of emotion expressions. Developmental Psychology, 37(6), 826. Doi: $10.1037 / 0012-1649.37 .6 .826$

Moses, L. J., Baldwin, D. A., Rosicky, J. G., \& Tidball, G. (2001). Evidence for referential understanding in the emotions domain at twelve and eighteen months. Child Development, 72(3), 718-735. Doi: 10.1111/1467-8624.00311

Müller, U., Liebermann-Finestone, D. P., Carpendale, J. I., Hammond, S. I., \& Bibok, M. B. (2012). Knowing minds, controlling actions: The developmental relations between theory of mind and executive function from 2 to 4 years of age. Journal of Experimental Child Psychology, 111(2), 331-348. Doi: 10.1016/j.jecp.2011.08.014

Nordmeyer, A. E., \& Frank, M. C. (2014). The role of context in young children's comprehension of negation. Journal of Memory and Language, 77, 25-39. Doi: 10.1016/j.jml.2014.08.002

Palmquist, C. M., Burns, H. E., \& Jaswal, V. K. (2012). Pointing disrupts preschoolers' ability to discriminate between knowledgeable and ignorant informants. Cognitive Development, 27(1),54-63. Doi:10.1016/j.cogdev.2011.07.002

Palmquist, C. M., \& Jaswal, V. K. (2012). Preschoolers expect pointers (even ignorant ones) to be knowledgeable. Psychological Science, 23(3), 230-231. Doi: $10.1177 / 0956797611427043$ 
Palmquist, C. M., Kondrad, R. L., \& Norris, M. N. (2018). Follow my point? Preschoolers' expectations about veridicality disrupt their understanding of deceptive points. Cognitive Development, 48, 190-202. Doi: 10.1016/j.cogdev.2018.08.009

Pea, R. (1980). The development of negation in early child language. In D. R. Olson (Ed.), The social foundations of language \& thought: Essays in honor of Jerome S. Bruner (edn, pp. 156 - 186). New York: W.W. Norton

Pexman, P. M. (2008). It's fascinating research: The cognition of verbal irony. Current Directions in Psychological Science, 17(4), 286-290. Doi: 10.1111/j.1467-8721.2008.00591.x

Phillips, A. T., Wellman, H. M., \& Spelke, E. S. (2002). Infants' ability to connect gaze and emotional expression to intentional action. Cognition, 85(1), 53-78. Doi: 10.1016/S0010-0277(02)00073-2

R Core Team. (2017). R: A language and environment for statistical computing. Vienna, Austria: R Foundation for Statistical Computing. Retrieved from https://www.R-project.org/ 
Repacholi, B. M. (1998). Infants' use of attentional cues to identify the referent of another person's emotional expression. Developmental Psychology, 34(5), 1017. Doi: $10.1037 / 0012-1649.34 .5 .1017$

Scherer, K. R., \& Ellgring, H. (2007). Multimodal expression of emotion: Affect programs or componential appraisal patterns? Emotion, 7(1), 158. Doi: 10.1037/1528-3542.7.1.158

Senju, A., \& Csibra, G. (2008). Gaze following in human infants depends on communicative signals. Current Biology, 18(9), 668-671. Doi: 10.1016/j.cub.2008.03.059

Senju, A., Csibra, G., \& Johnson, M. H. (2008). Understanding the referential nature of looking: Infants' preference for object-directed gaze. Cognition, 108(2), 303-319. Doi: 10.1016/j.cognition.2008.02.009

Smetana, J. G., Kochanska, G., \& Chuang, S. (2000). Mothers' conceptions of everyday rules for young toddlers: A longitudinal investigation. Merrill-Palmer Quarterly, 391-416.

Sorce, J. F., Emde, R. N., Campos, J. J., \& Klinnert, M. D. (1985). Maternal emotional signaling: Its effect on the visual cliff behavior of 1-year-olds. Developmental Psychology, 21(1), 195. Doi: 10.1037/0012-1649.21.1.195

Southgate, V., Chevallier, C., \& Csibra, G. (2010). Seventeen-month-olds appeal to false beliefs to interpret others' referential communication. Developmental Science, 13(6), 907-912. Doi: 10.1111/j.1467-7687.2009.00946.x 
Stengelin, R., Grueneisen, S., \& Tomasello, M. (2018). Why should I trust you? Investigating young children's spontaneous mistrust in potential deceivers. Cognitive Development, 48, 146-154. Doi: 10.1016/j.cogdev.2018.08.006

Tomasello, M. (2003). Constructing a language: A usage-based approach to child language acquisition.

Tomasello, M. (2008). Origins of human communication. Cambridge, MA: MIT Press.

Tomasello, M., Hare, B., Lehmann, H., \& Call, J. (2007). Reliance on head versus eyes in the gaze following of great apes and human infants: the cooperative eye hypothesis. Journal of Human Evolution, 52(3), 314-320. Doi: 10.1016/j.jhevol.2006.10.001

Walle, E. A., Reschke, P. J., Camras, L. A., \& Campos, J. J. (2017). Infant differential behavioral responding to discrete emotions. Emotion, 17(7), 1078. Doi:10.1037/emo0000307.

Wilson, D. (2013). Irony comprehension: A developmental perspective. Journal of Pragmatics, 59, 40-56. Doi: 10.1016/j.pragma.2012.09.016

Vanderbilt, K. E., Liu, D., \& Heyman, G. D. (2011). The development of distrust. Child Development, 82(5), 1372-1380. Doi: 10.1111/j.1467-8624.2011.01629.x 
Yurovsky, D., \& Frank, M. C. (2017). Beyond naïve cue combination: Salience and social cues in early word learning. Developmental Science, 20(2). Doi: 10.1111/desc.12349 